Presentación de caso

\section{Dermatosis inducida por el agua: queratodermia acuagénica, a propósito de un caso}

\author{
Camila Montoya, Liza M. Arias, Mónica Salazar, Rodrigo Restrepo
}

Programa de Dermatopatología, Universidad CES, Medellín, Colombia

La queratodermia acuagénica es una entidad benigna, caracterizada por producir pápulas blanquecinas o traslúcidas pocos segundos después del contacto con el agua. Se presenta el caso de una paciente de 16 años de edad con aparición de múltiples pápulas confluentes y asintomáticas en ambas palmas al contacto con el agua, que desaparecían luego del secado. En el estudio de histopatología se observó dilatación de los conductos ecrinos y cambios en el estrato córneo.

Esta rara condición de etiología desconocida se ha relacionado con disfunción neuronal, alteraciones de las glándulas ecrinas y, más recientemente, con alteraciones en las acuaporinas. Se puede diagnosticar con una prueba semiológica sencilla llamada 'la mano en el balde'; la sospecha clínica es fundamental para hacer el diagnóstico, ya que los hallazgos histopatológicos pueden ser sutiles e inespecíficos. El tratamiento tópico incluye mecanismos de barrera y la toxina botulínica.

Palabras clave: enfermedades de la piel; queratodermia palmoplantar; agua; informes de casos.

Water-induced dermatosis: Aquagenic keratoderma. A case report

Aquagenic keratoderma is a benign entity that is characterized by producing whitish or translucent papules a few seconds after contact with water.

We present the case of a 16-year-old patient with multiple asymptomatic confluent papules that appeared on both hand palms after contact with water and which disappeared after drying. The histopathological findings in a skin biopsy after water exposure showed changes in the superficial layers of the stratum corneum and dilatation of sweat gland ducts.

This entity of unknown etiology has been related to neuronal and eccrine gland dysfunction. Recently it has been associated with alterations of aquaporins. The "hand-in-the-bucket" sign is a simple useful clinical tool for diagnosis, as histopathological findings may be nonspecific. Topical treatments include barrier mechanisms and botulinum toxin.

Keywords: Skin diseases; keratoderma, palmoplantar; water; case reports.

La queratodermia acuagénica es una entidad poco frecuente descrita por primera vez como 'acroqueratoderma papular translúcida transitoria' por English y McCollough en 1996 (1); se caracteriza por producir pápulas blanquecinas o traslúcidas pocos segundos después del contacto con el agua (2-4).

La finalidad de esta comunicación es agregar un caso más a la literatura de esta enfermedad inusual que, hasta donde lo hemos podido comprobar, es el primer caso reportado en Colombia. Asimismo, hacer una revisión breve de la literatura científica.

\section{Caso clínico}

Se presenta el caso de una paciente de 16 años de edad con un cuadro clínico de un año de evolución, caracterizado por la aparición de múltiples pápulas traslúcidas y confluentes en ambas palmas luego de pocos minutos de tener contacto con el agua (figuras 1 y 2). Las lesiones eran asintomáticas y desaparecían por completo después del secado mecánico de las manos (figura 3). La paciente refirió no haber consumido ningún medicamento y negó tener enfermedades de base.

Se le tomó una biopsia de piel de la palma inmediatamente después del contacto con el agua. El estudio histopatológico reveló la presencia de piel distal (acral) con hiperqueratosis y áreas basofílicas en el estrato córneo, asociadas con dilatación de los conductos ecrinos (figura 4). Con estos hallazgos y los antecedentes clínicos, se hizo el diagnóstico de queratodermia acuagénica. 

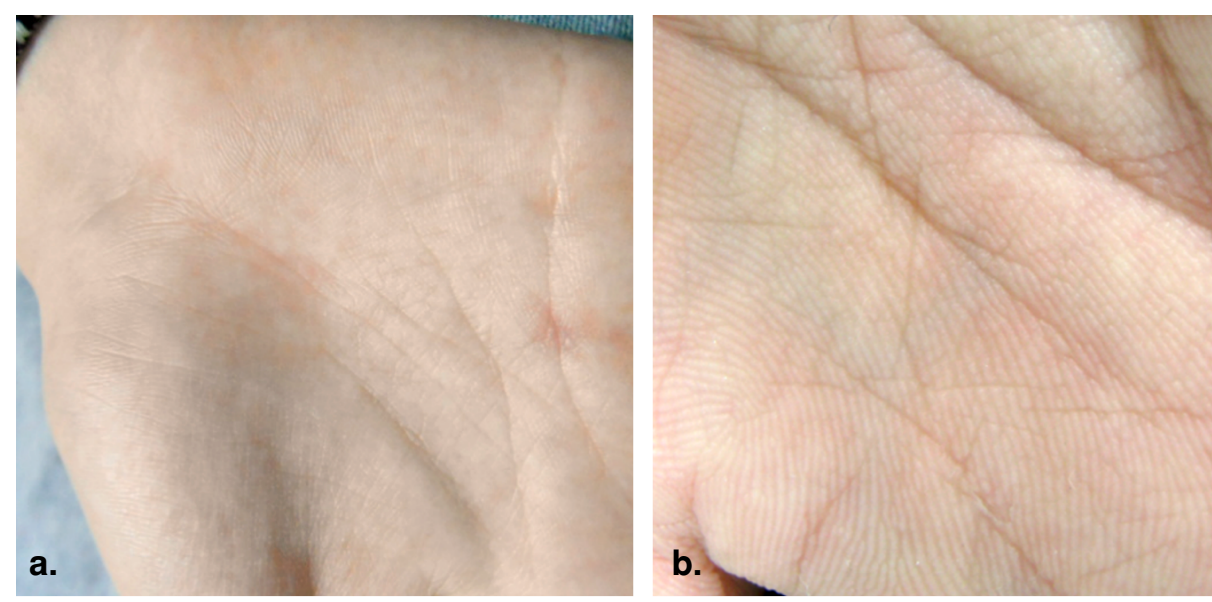

Figura 1. Palma de la mano derecha: a) antes del contacto con el agua: discreta prominencia de los surcos y crestas; b) acercamiento minutos después del secado: ausencia de lesiones palmares
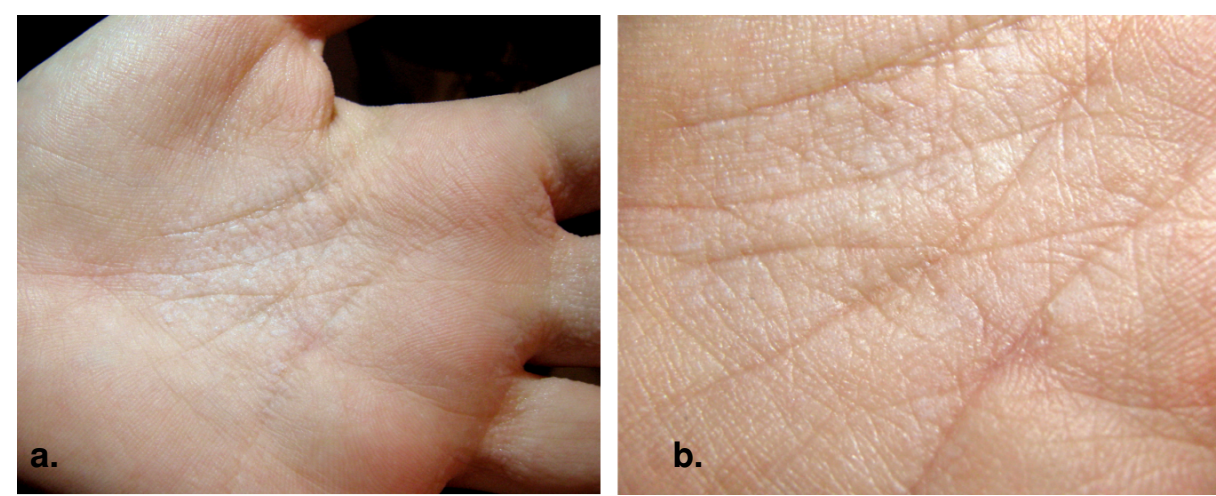

Figura 2. Palma de la mano izquierda: a) después del contacto con el agua se observan pápulas translúcidas confluentes con tendencia a la formación de placas; b) acercamiento de las mismas lesiones

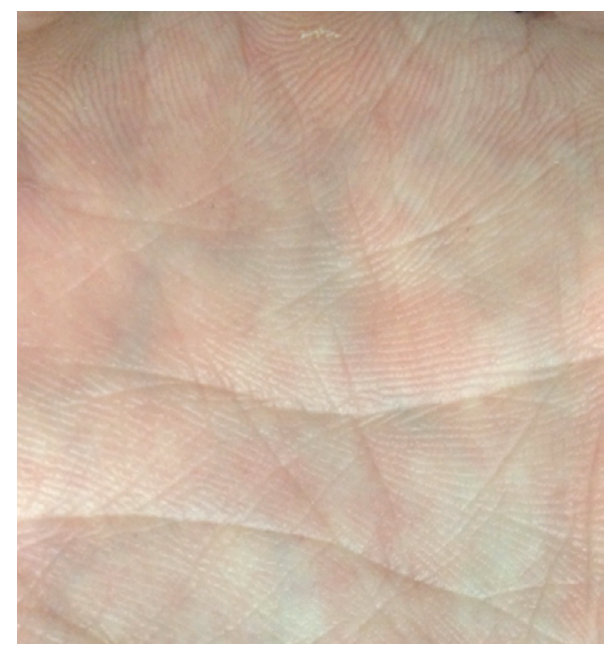

Figura 3. Palma de la mano después del secado: ausencia de lesiones palmares 


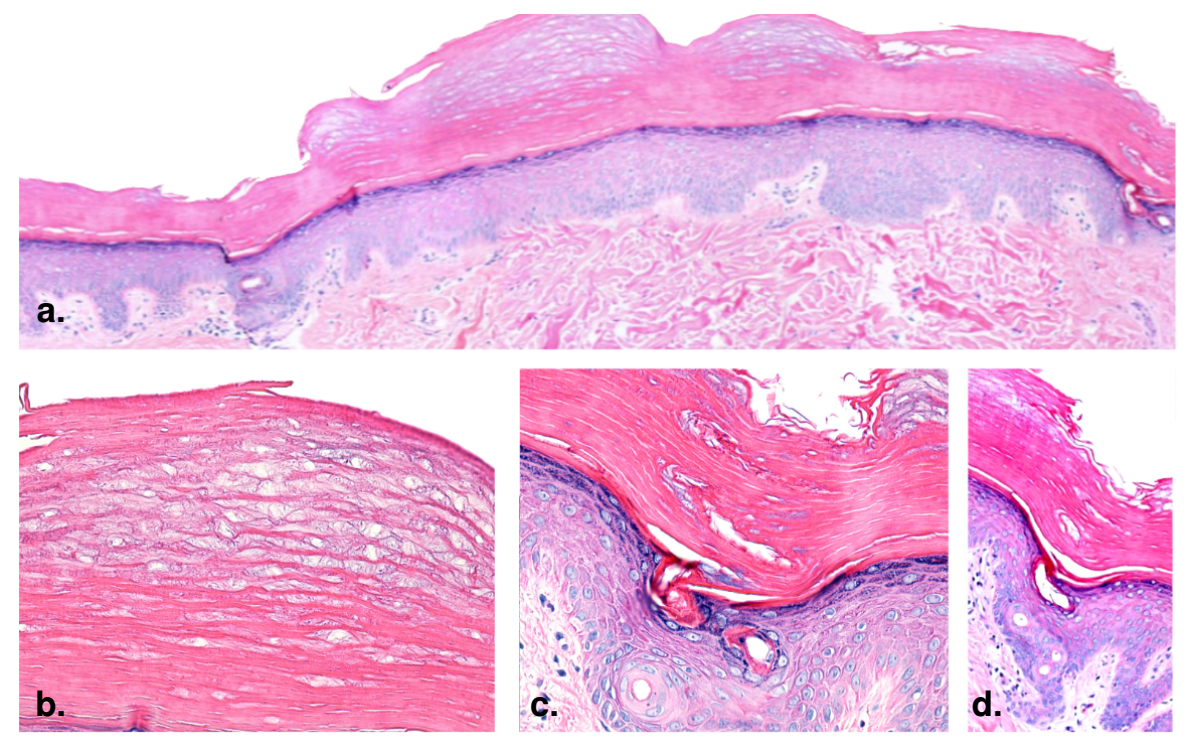

Figura 4. a) Vista panorámica: alteración de la tinción del estrato córneo y dilatación focal de los conductos ecrinos. Hematoxilina y eosina, 2X. b) Tinción basofílica del estrato córneo. Hematoxilina y eosina, 40X. c) y d) Dilatación de los conductos ecrinos. Hematoxilina y eosina, 10X y 4X

\section{Discusión}

La queratodermia acuagénica se describió inicialmente en 1996 (1) y, aunque es infrecuente, el número de casos reportados ha ido en aumento. Esto lleva a pensar que esta entidad ha sido subdiagnosticada y su incidencia es mayor de la esperada. Debido a su particular relación con el agua, la sospecha clínica es fundamental para el diagnóstico. Una prueba semiológica sencilla llamada 'la mano en el balde' (5) y los antecedentes clínicos permiten orientar el diagnóstico.

Las mujeres se ven más frecuentemente afectadas. El rango etario es variable, sin embargo, su presentación en la infancia es rara (6). Usualmente, compromete las palmas, los dedos de las manos $y$, menos frecuentemente, las plantas de los pies (7); el compromiso bilateral es más característico. Se han reportados casos con características atípicas, como distribución unilateral (8) y presentación en el sexo masculino, y de localizaciones inusuales, como el dorso de las manos (9) y el dorso nasal (10). La mayoría de los casos son esporádicos, aunque se han descrito casos familiares (11).

La característica clínica principal es la inducción de lesiones después del contacto con el agua durante algunos minutos (en promedio, tres minutos) (10). Los pacientes presentan múltiples pápulas blanquecinas o translúcidas que pueden confluir, dando lugar a placas edematosas de aspecto macerado que, habitualmente, se ubican en el borde marginal de las palmas y desaparecen minutos después del secado (3). Por lo general, estas lesiones son asintomáticas y, con menor frecuencia, se asocian a ardor, dolor o prurito (3). La hiperhidrosis es un hallazgo frecuente en estos pacientes y, al mismo tiempo, actúa como un factor desencadenante $(3,7,10)$.

La etiología de la queratodermia acuagénica sigue siendo desconocida, pero se han postulado varias hipótesis sobre causas probables, entre ellas, disfunción neuronal, hiperhidrosis, alteración de la osmolaridad y la concentración de sal en el estrato córneo, disfunción de los conductos 
ecrinos y obstrucción del ostium (7). Más recientemente, se ha descrito su relación con alteraciones en las acuaporinas, específicamente la AQ-5 (12). La asociación descrita entre queratodermia acuagénica y fibrosis quística (13) podría sugerir que existe una fuerte relación entre la exposición a concentraciones anormales de sal en la epidermis y la patogenia de esta entidad (14). Esto también podría explicar la aparición de lesiones similares que se relacionan con el consumo de inhibidores de la COX-2 (15), aminoglucósidos y espironolactona (10).

La biopsia siempre debe tomarse después de la exposición al agua; sin embargo, los hallazgos histopatológicos pueden ser sutiles e, incluso, estar ausentes (16), por lo cual pueden pasar desapercibidos fuera del contexto clínico. La dilatación de los conductos ecrinos es el hallazgo más frecuente, tal como se observó en este caso, pero también pueden verse alteraciones de los acinos ecrinos, con hiperplasia, aspecto granular del citoplasma $(16,17)$, cambios en las células claras $(7)$, núcleos picnóticos y eosinofilia. El estrato córneo puede presentar hiperqueratosis, edema, tinción anormal o pálida y dilatación de los acrosiringios $(7,17)$. Con menor frecuencia, se ha descrito hiperplasia de los acrosiringios, incluso con formación de proyecciones papilares (10), espongiosis de los conductos ecrinos, acantosis de la epidermis e infiltrado linfocitario dérmico (16).

El principal diagnóstico diferencial clínico e histológico es la acroqueratodermia pápulo-traslúcida hereditaria, la cual se caracteriza por lesiones palmares permanentes que se intensifican al entrar en contacto con el agua, como ocurre en la queratodermia acuagénica, pero, a diferencia de esta, no desaparecen al secarse la piel. Histológicamente, se presenta alteración del estrato córneo con hiperqueratosis, hipergranulosis y acantosis, en ausencia de compromiso de los conductos y acinos ecrinos (5).

Aunque la queratodermia acuagénica es una entidad benigna, puede alterar la calidad de vida del paciente. El tratamiento incluye la utilización de mecanismos de barrera, como el sulfuro de petróleo y el cloruro de aluminio. Existen casos reportados con buena respuesta a la toxina botulínica (8) y a la iontoforesis (18), lo que estaría a favor del rol principal de las glándulas sudoríparas en la patogenia de esta enfermedad.

En conclusión, esta entidad es una dermatosis transitoria inducida por el agua, de etiología desconocida, que puede diagnosticarse con una prueba semiológica sencilla, siendo la sospecha clínica fundamental para su diagnóstico. La biopsia debe tomarse únicamente después de que el paciente haya tenido contacto con el agua (17). Dado que los hallazgos histopatológicos pueden ser sutiles o inespecíficos, los antecedentes y la correlación clínico-patológica son imprescindibles para el diagnóstico.

\section{Referencias}

1. English JC, McCollough ML. Transient reactive papulotranslucent acrokeratoderma. J Am Acad Dermatol. 1996;34:686-7. https://doi.org/10.1016/S0190-9622(96)80087-9

2. Tchernev G, Semkova K, Cardoso JC, Ananiev JJ, Wollina U. Aquagenic keratoderma. Two new case reports and a new hypothesis. Indian Dermatol Online J. 2014;5:30-3. https://doi.org/10.4103/2229-5178.126025

3. Ertürk-Özdemir E, Özcan D, Seçkin D. Acquired aquagenic syringeal acrokeratoderma: A case series of 10 patients. Australas J Dermatol. 2015;56:e43-5. https://doi.org/10.1111/ajd.12122

4. Lafuente-Urrez RF, Pérez-Pelegay J, Corredera-Carrión C. Queratodermia acuagénica: aportación de dos nuevos casos. Piel. 2014;29:60-2. https://doi.org/10.1016/j.piel.2013.06.003 
5. Yan AC, Aasi SZ, Alms WJ, James WD, Heymann WR, Paller AS, et al. Aquagenic palmoplantar keratoderma. J Am Acad Dermatol. 2001;44:696-9.

https://doi.org/10.1067/mjd.2001.113479

6. Qing X. Aquagenic acrokeratoderma: Case report with no involvement of the palms. Int J Dermatol. 2012;51:1388-403. https://doi.org/10.1111/j.1365-4632.2010.04803.x

7. Rongioletti F, Tomasini C, Crovato F, Marchesi L. Aquagenic (pseudo) keratoderma: A clinical series with new pathological insights. Br J Dermatol. 2012;167:575-82. https://doi.org/10.1111/j.1365-2133.2012.11003.x

8. Houle M-C, Al Dhaybi R, Benohanian A. Unilateral aquagenic keratoderma treated with botulinum toxin A. J Dermatol Case Rep. 2010;4:1-5. https://doi.org/10.3315/idcr.2010.1045

9. Pardo J, Sánchez-Motilla JM, Latasa JM. Queratodermia acuagénica de características atípicas. Actas Dermosifiliogr. 2005;96:540-2. https://doi.org/10.1016/S0001-7310(05)73130-6

10. Peña-Romero AG, Toussaint-Caire S, Charli-Joseph Y, Dela Barreda-Becerril F, DomínguezCherit J. From classical to unusual: Report on 5 cases of transient reactive aquagenic pseudokeratoderma and review of the literature. Am J Dermatopathol. 2017;39:935-42. https://doi.org/10.1097/DAD.0000000000000815

11. Nazik H, Nazik S, Çoban FG, Demir B. Sporadic and familial cases of aquagenic keratoderma. J Dermatol Case Rep. 2016;10:10-3. https://doi.org/10.3315/jdcr.2016.1223

12. Kabashima K, Shimauchi T, Kobayashi M, Fukamachi S, Kawakami C, Ogata M, et al. Aberrant aquaporin 5 expression in the sweat gland in aquagenic wrinkling of the palms. $J$ Am Acad Dermatol. 2008;59:S28-32. https://doi.org/10.1016/j.jaad.2008.04.023

13. Garçon-Michel N, Roguedas-Contios A-M, Rault G, Le Bihan J, Ramel S, Revert K, et al. Frequency of aquagenic palmoplantar keratoderma in cystic fibrosis: A new sign of cystic fibrosis? Br J Dermatol. 2010;163:162-6. https://doi.org/10.1111/j.1365-2133.2010.09764.x

14. Katz KA, Yan AC, Turner ML. Aquagenic wrinkling of the palms in patients with cystic fibrosis homozygous for the $\Delta$ F508 CFTR mutation. Arch Dermatol. 2005;141:621-4. https://doi.org/10.1001/archderm.141.5.621

15. Gündüz Ö, Özsaraç K, Ercin ME. Aquagenic palmar wrinkling induced by combined use of salazopyrin and indomethacin. Case Rep Dermatol. 2013;5:21-6. https://doi.org/10.1159/000346744

16. Pastor MA, González L, Kilmurray L, Bautista P, López A, Puig AM. Queratodermia acuagénica. Actas Dermosifiliogr. 2008;99:399-406. https://doi.org/10.1016/S0001-7310(08)74700-8

17. Baldwin BT, Prakash A, Fenske NA, Messina JL. Aquagenic syringeal acrokeratoderma: Report of a case with histologic findings. J Am Acad Dermatol. 2006;54:899-902. https://doi.org/10.1016/j.jaad.2005.09.007

18. Errichetti E, Piccirillo A. Aquagenic keratoderma treated with tap water iontophoresis. Indian J Dermatol. 2015;60:212. https://doi.org/10.4103/0019-5154.152568 